\title{
INFLUENCE OF HEMA CONTENT ON THE MECHANICAL AND BONDING PROPERTIES OF EXPERIMENTAL HEMA- ADDED GLASS IONOMER CEMENTS
}

\author{
Ho-Nam LIM', Seong-Hwan KIM ${ }^{2}$, Bin YU ${ }^{3}$, Yong-Keun LEE ${ }^{4}$
}

\begin{abstract}
1- DDS, PhD, Professor, Department of Dental Materials and Center for Dental Materials, School of Dentistry, Kyung Hee University, Seoul, Korea. 2- PhD student, Department of Dental Materials and Center for Dental Materials, School of Dentistry, Kyung Hee University, Seoul, Korea.

3- PhD student, Department of Dental Biomaterials and Dental Research Institute, School of Dentistry, Seoul National University, Seoul, Korea. 4- DDS, PhD, Professor, Department of Dental Biomaterials and Dental Research Institute, School of Dentistry, Seoul National University, Seoul, Korea.
\end{abstract}

Corresponding address: Yong-Keun Lee, DDS, PhD - Department of Dental Biomaterials Science, School of Dentistry, Seoul National University, 28 Yeongeon-dong, Jongro-gu, Seoul, Korea - Phone: 82-2-740-8693 - Fax: 82-2-740-8694 - e-mail: ykleedm@ snu.ac.kr

Received: October 21, 2008 - Modification: February 12, 2009 - Accepted: February 13, 2009

\begin{abstract}
$T_{\text {he }}$

he purpose of this study was to determine the influence of incrementally added uncured HEMA in experimental HEMA-added glass ionomer cement (HAGICs) on the mechanical and shear bond strength (SBS) of these materials. Increasing contents of uncured HEMA (10-50 wt.\%) were added to a commercial glass ionomer cement liquid (Fuji II, GC, Japan), and the compressive and diametral tensile strengths of the resulting HAGICs were measured. The SBS to non-precious alloy, precious alloy, enamel and dentin was also determined after these surfaces were subjected to either airborne-particle abrasion (Aa) or SiC abrasive paper grinding (Sp). Both strength properties of the HAGICs first increased and then decreased as the HEMA content increased, with a maximum value obtained when the HEMA content was $20 \%$ for the compressive strength and $40 \%$ for the tensile strength. The SBS was influenced by the HEMA content, the surface treatment, and the type of bonding surface $(\mathrm{p}<0.05)$. These results suggest that addition of an appropriate amount of HEMA to glass ionomer cement would increase diametral tensile strength as well as bond strength to alloys and teeth. These results also confirm that the optimal HEMA content ranged from 20 to $40 \%$ within the limitations of this experimental condition.
\end{abstract}

Key words: Glass ionomer cement. HEMA. Compressive strength. Diametral tensile strength. Shear bond strength.

\section{INTRODUCTION}

Glass ionomer cement (GIC) was developed by Wilson and Kent in the 1970s, and shows clinical advantages such as fluoride release, bonding to teeth and alloys, and physiological compatibility with teeth. Therefore, this cement has been widely used in varied fields of dental practice $^{5,13,22}$. However, several shortcomings of this material such as insufficient mechanical properties, decreased translucency when contaminated by moisture before setting and desiccation under dry conditions have been demonstrated $^{26}$. Several studies have been performed to address these problems, and can be assigned to two categories $^{22}$. The first category of studies investigated the improvement of the mechanical properties of GICs by adding tough and ductile fillers such as aluminosilicate glass ${ }^{20}$, metal powder mixed with glass ${ }^{24}$, and reinforcing glass fiber ${ }^{12}$. The second category of studies investigated the modification of the matrix or the matrix interface by either increasing the molecular weight of the polyacrylic acid in the cement liquid $^{20}$, or by adding maleic acid copolymer, acrylic-itaconic acid copolymer, bisphenol glycidylmethacrylate (BisGMA), or triethyleneglycol dimethacrylate (TEGDMA) to the liquid ${ }^{13}$.

In the early 1990 s, a resin monomer named 2hydroxyethylmethacrylate (HEMA) was added to GIC to fabricate a modified cement called hybrid ionomer or resinmodified glass ionomer cement (RMGIC) ${ }^{4}$. The first commercial RMGIC was patented in $1989^{14}$. It was claimed to contain a photocurable ionomer, reactive powder and water, and to undergo both a conventional setting reaction and a photocuring reaction. This cement system was also claimed to provide a longer working time because it could be cured on demand by exposure to an appropriate source of radiant energy. This new type of cement has been modified in varied directions and is widely used in clinic. The effect of replacing the water with methanol, 50/50 water/methanol or 50/50 water/HEMA on a water-activated glass polyacrylic 
dental cement has also been studied ${ }^{1}$. RMGIC sets through the complicated interaction of polymerization reactions and acid-base reactions between glass particles and polyacrylic acid, and also between glass particles and acidic resin monomers ${ }^{8,10}$. In the setting process, the intensity of the acidbase reaction decreases as the amount of resin components increases and also decreases through the decreased diffusion of ions as the cross-linking of resins proceeds. Since the polymerization reaction is influenced by the polarity of the acid-base matrix, a polar polyacid in the cement liquid can easily change the efficacy of the polymerization reactions, namely the degree of polymerization ${ }^{17}$. The complicated chemical reactions in RMGIC are practically impossible to control because the acid-base reaction and resin polymerization occur simultaneously, and the exact role of HEMA in the setting reaction had not yet been defined. However, a previous study found that HEMA can enhance the early mechanical properties and working characteristics of a $\mathrm{RMGIC}^{25}$.

Several studies on RMGIC have been performed. Mathis and Ferracane ${ }^{13}$ found that the mechanical properties of a RMGIC measured after setting for $1 \mathrm{~h}$ were generally higher than those of a conventional GI, while the compressive strength of the RMGIC after setting for $24 \mathrm{~h}$ was lower than that of the GIC. The bond strength of the RMGIC to tooth was similar to that of the $\mathrm{GIC}^{13}$. In another study, the early handling performance and mechanical properties of a RMGIC were better than those of a conventional GIC, however, the mechanical properties after final setting were similar for both materials ${ }^{25}$. Other studies reported that the mechanical properties and bond strength of RMGIC were better than those of a conventional $\mathrm{GIC}^{23}$. However, none of these studies identified generally applicable conclusions that considered both the compositional differences and experimental conditions. Nonetheless, Cattani-Lorente ${ }^{5}$ have reported that although the mechanical properties of HEMAsupplemented RMGICs are generally regarded as better than those of conventional GICs, the mechanical properties of these materials changed after long-term immersion in water, and several properties of the RMGIC were poorer than those of the conventional GI. These decreased long-term properties of the RMGIC have been attributed to the hydrolysis and degradation of HEMA ${ }^{27}$.

Although several studies on the function and effect of HEMA in RMGIC have been reported, most of these studies were based on commercial materials or on limited numbers of fixed HEMA contents. Therefore, the influence of HEMA content on the properties of RMGIC has been investigated qualitatively, not quantitatively and systematically. As a method for a clear determination of the effect of uncured HEMA added to GIC, the influence of polymerization of HEMA could be excluded. Based on this experimental result, the influence of HEMA polymerization on RMGIC would be decided afterwards. However, to the best of our knowledge, there are no studies on the effect of uncured HEMA on the mechanical and bonding properties of this type of material, except for the study by Anstice and Nicholson ${ }^{1}$. Among the studies that investigated the bonding performance of RMGIC, one found that the bond strength to airborne particle-abraded dentin was lower than that to dentin ground with $\mathrm{SiC}$ abrasive papers ${ }^{21}$. However, another study reported that the bond strengths to airborne particleabraded dentin and enamel were higher than those to enamel and dentin whose surfaces were ground with $\mathrm{SiC}$ abrasive papers ${ }^{16}$. Thus, there is no consensus as to which surface treatment method results in higher bond strength.

The purpose of the present study was to determine the influence of the incrementally added uncured HEMA in an experimental HEMA-added glass ionomer cement (HAGIC) on the mechanical and shear bond strength (SBS). The bonding surfaces were dental alloys and teeth treated with airborne-particle abrasion or grinding with \#1000 SiC paper. The tested null hypotheses were that there are no significant differences in the mechanical properties of the experimental HAGICs with increasing HEMA contents, and that there are no significant differences in the SBS of these HAGICs to dental alloys and teeth as a function of HEMA content.

\section{MATERIAL AND METHODS}

\section{Materials}

Powder and liquid of Fuji II glass ionomer cement (Shade No. 21, Lot No. 0612071, GC Corp., Tokyo, Japan) were used as starting compositions of the experimental HAGICs. Fuji II liquid (GC Corp.) and HEMA (Junsei, Tokyo, Japan) were thoroughly mixed as indicated in Table 1 and were stored in a cold and dark environment. Conventional Fuji II filling glass ionomer cement was used as a control group. For the bonding substrates, a nickel-chrome based alloy (Unimetal-II; Shofu, Kyoto, Japan) was used as a nonprecious alloy and an ADA type 2 alloy (Cast-2; Alphadent, Gyeonggi, Korea) was used as a precious alloy. Intact human enamel and dentin were also used.

\section{Compressive and Diametral Tensile Strengths}

Cylindrical specimens (6 mm height x $4 \mathrm{~mm}$ diameter) were fabricated to measure the compressive strength, and disk-shaped specimens ( $2 \mathrm{~mm}$ thickness $\mathrm{x} 4 \mathrm{~mm}$ diameter) were fabricated to measure the diametral tensile strength. Teflon molds with the same sizes of the specimens and a mold wall thickness of $2 \mathrm{~mm}$ were used to prepare the specimens. The powder and liquid were mixed following the manufacturer's instructions and were poured into the molds. The open end of the mold was covered with a celluloid matrix strip (Striproll; KerrHawe, Bioggio, Switzerland). Specimens were removed from the mold after 15 min from the start of mixing by using a wooden stick and were immersed in $37^{\circ} \mathrm{C}$ distilled water for $24 \mathrm{~h}$. Eight specimens were prepared for each group.

The mechanical properties were measured in a universal testing machine (Instron 4467, Instron Co., Canton, MA, USA) with a crosshead speed of $1 \mathrm{~mm} / \mathrm{min}$. The compressive and diametral tensile strengths were calculated using the following equations in $\mathrm{MPa}$ units. Compressive strength: $K=4 F / T d^{2}$; diametral tensile strength: $\sigma=2 F / T \pi d t$, where $F$ 
TABLE 1- Preparation of the liquid component to produce experimental HAGICs

\begin{tabular}{lcc}
\hline Code & Commercial liquid ${ }^{\mathrm{a}}(\mathbf{g})$ & HEMA $^{\mathrm{b}}(\mathbf{g})$ \\
\hline Control & 5.0 & 0 \\
10\% HEMA & 4.5 & 0.5 \\
20\% HEMA & 4.0 & 1.0 \\
30\% HEMA & 3.5 & 1.5 \\
40\% HEMA & 3.0 & 2.0 \\
$50 \%$ HEMA & 2.5 & 2.5 \\
\hline
\end{tabular}

Control = Conventional filling glass ionomer cement (Fuji II, GC Corp., Tokyo, Japan). a = Liquid component of Fuji II (GC Corp.); b = 2-hydroxyethylmethacrylate (Lot No. 5K6113; JUNSEI, Japan).

indicates load $(\mathrm{N}), d$ indicates the diameter of the specimen $(\mathrm{mm})$, and $t$ indicates the specimen thickness $(\mathrm{mm})$.

\section{Shear Bond Strength}

Nonprecious and precious alloy specimens were fabricated by casting in a plate form of $10 \times 10 \times 2 \mathrm{~mm}^{3}$ and then embedded in embedding resin (Polycoat; Aekyung, Chungnam, Korea). Freshly extracted sound teeth were selected and root portion of the teeth were removed. Thereafter, each tooth was embedded in embedding resin (Polycoat, Aekyung), leaving exposed the bonding surface. Tooth surface for bonding was selected in such a way that the available bonding surface was at least $5 \mathrm{~mm}$ in diameter. The enamel test surfaces were obtained from the labial surface of anterior teeth, while the dentin test surfaces were obtained from the buccal or lingual surface of molars. Embedded alloys and teeth were ground flat with wet \#600 SiC paper (3M, St. Paul, MN, USA) and were randomly divided into two groups. For the airborne particle abrasion (Aa) group, airborne-particle abrasion was performed with $50 \mu \mathrm{m}$ aluminum oxide particles under $5.51 \times 10^{5} \mathrm{~Pa}$ with a sandblasting machine (Basic master No.2946; Renfert, Hilzingen, Germany). The operation time was $6 \mathrm{~s}$ and the operation distance was $10 \mathrm{~mm}$. For the $\mathrm{SiC}$ abrasive paper grinding (Sp) group, grinding with \#1000 SiC paper was additionally performed to obtain smoother surfaces, which was supposed to make clear difference in bonding performance.

A Teflon mold with an inner diameter of $4 \mathrm{~mm}$, thickness of $2 \mathrm{~mm}$ and wall thickness of $2 \mathrm{~mm}$ was overlaid on the prepared bonding surface ${ }^{15}$. The experimental HAGIC was mixed and poured into the mold. A celluloid matrix strip (Striproll) was placed over the opening of the mold. The specimens were then securely fixed with a clamp. The mold was removed $15 \mathrm{~min}$ after the start of mixing and the specimens were then immersed in $37^{\circ} \mathrm{C}$ distilled water for $24 \mathrm{~h}$. Mold was removed with a scalpel under the condition that the set material was pressed with a wooden stick. Eight specimens were prepared for each group, totalizing 80 specimens.

SBS was measured in the universal testing machine (Instron 4467) with a crosshead speed of $1 \mathrm{~mm} / \mathrm{min}$. Shear bond strength was calculated in MPa units using the following equation: Shear bond strength $T=4 F / T T d^{2}$, where $F$ indicates load $(\mathrm{N})$ and $d$ indicates the diameter of the bonded surface $(\mathrm{mm})$.

\section{Scanning Electron Microscopic and Atomic Force Microscopic Analyses of the Bonding Surfaces}

To determine the characteristics of the bonding surfaces treated with either Aa or Sp, scanning electron microscope (SEM: Stereoscan 440; Leica, Cambridge, England) and an atomic force microscope (AFM: AutoProbe CP Research System; Thermo Fisher Scientific, Waltham, MA, USA) were used. For the SEM, the accelerating voltage was 20 $\mathrm{kV}$, the vacuum level was $1.33 \times 10^{-4} \mathrm{~Pa}$, the working distance was $11 \mathrm{~mm}$, and the surface topography was observed at $\times 5000$ magnification. For the AFM, the resonance frequency was $320 \mathrm{kHz}$ and the cantilever tip height was $12.5 \mu \mathrm{m}$. The average roughness value $(\mathrm{Ra})$ was determined with the results from the AFM.

\section{Statistical Analysis}

Means and standard deviations were calculated for each measurement. To determine the influence of HEMA content on the compressive and diametral tensile strengths, a oneway ANOVA with a significance level of 0.05 (SPSS 12.0; SPSS Co., Chicago, IL, USA) was performed. To determine the influence of HEMA content and surface treatment on the SBS, two-way ANOVA was performed for each bonding surface. The means were compared with Scheffe's multiplecomparison tests $(\mathrm{p}<0.05)$.

\section{RESULTS}

\section{Compressive and Diametral Tensile Strengths}

The compressive and diametral tensile strengths of the experimental HAGICs with different HEMA contents are presented in Figure 1. Based on one-way ANOVA, both strengths were significantly influenced by the HEMA content $(\mathrm{p}<0.05)$. There was no significant difference in the compressive strength among the control group and the $10 \%$, $20 \%$ and 30\% HEMA groups based on Scheffe's multiplecomparison test $(\mathrm{p}>0.05)$. When the HEMA content was higher than $30 \%$, the compressive strength decreased 
significantly $(\mathrm{p}<0.05)$, but there was no significant difference in the compressive strength among the $30 \%, 40 \%$ and $50 \%$ HEMA groups. HEMA addition showed trends as firstly increase and then decrease of the diametral tensile strength as the HEMA content increased, and the maximum value was observed when the HEMA content was $40 \%$. Based on Scheffe's multiple-comparison test, the HEMA-added groups presented significantly higher tensile strength than
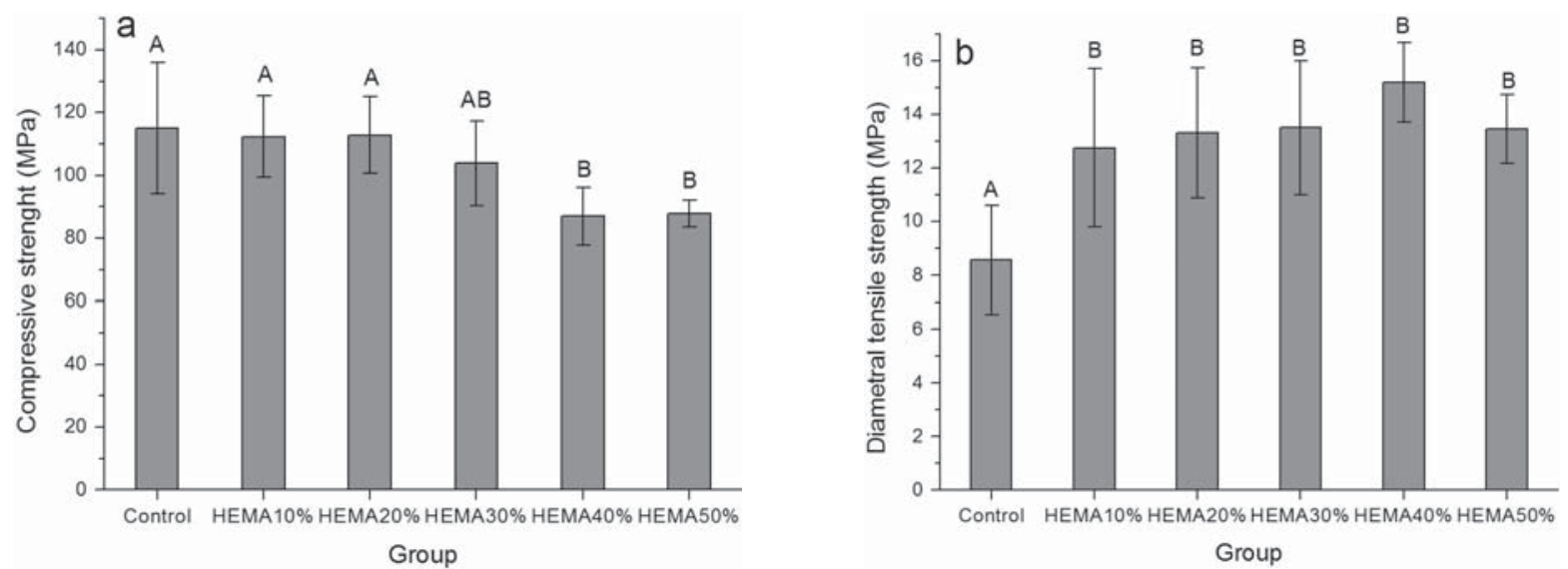

FIGURE 1- Compressive strength (a) and diametral tensile strength (b) of experimental HEMA-added glass ionomer cements. Groups marked with the same letters were not significantly different $(p>0.05)$
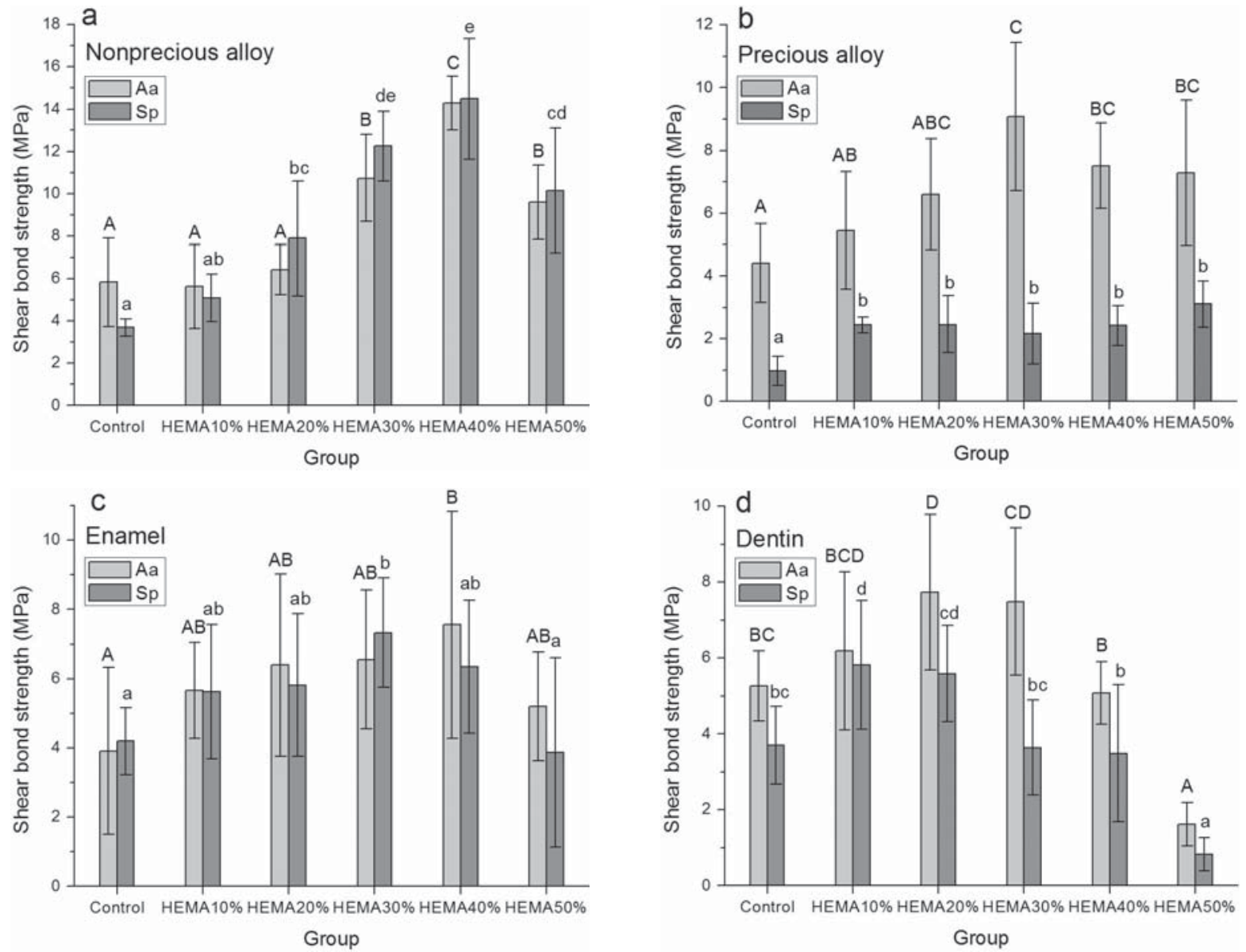

FIGURE 2- Shear bond strength of experimental HEMA-added glass ionomer cement to a nonprecious alloy (a), a precious alloy (b), enamel (c), and dentin (d). Aa groups marked with the same uppercase letters were not significantly different $(p>0.05)$. Sp groups marked with the same lowercase letters were not significantly different $(p>0.05)$ 

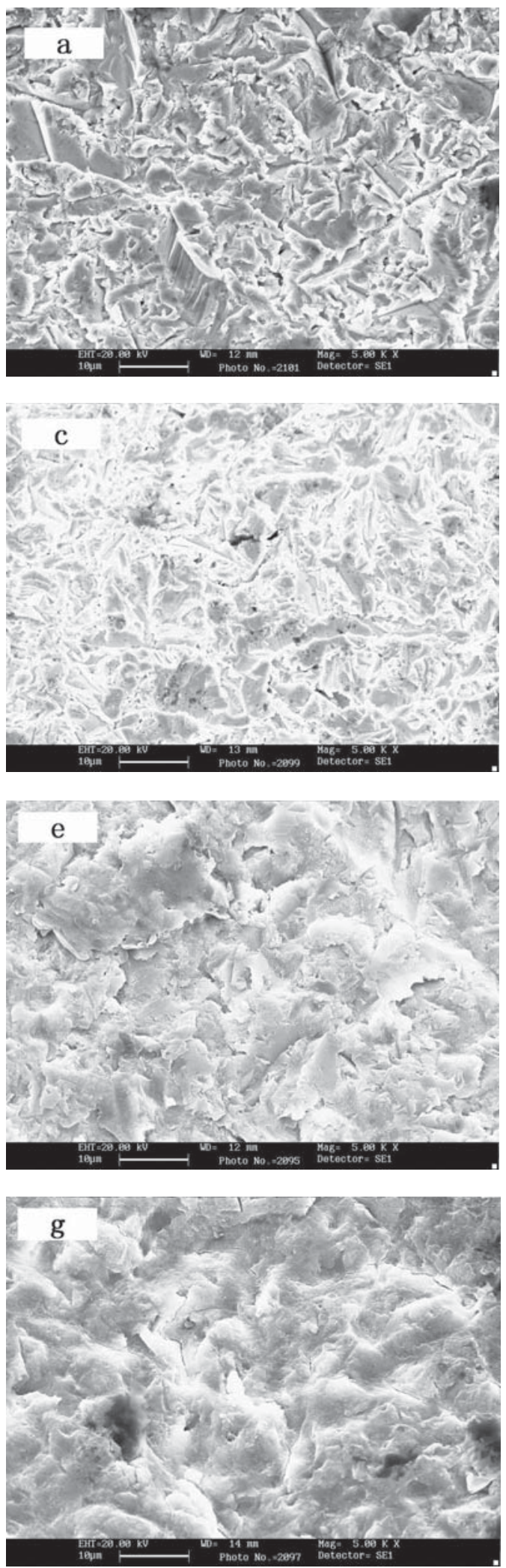
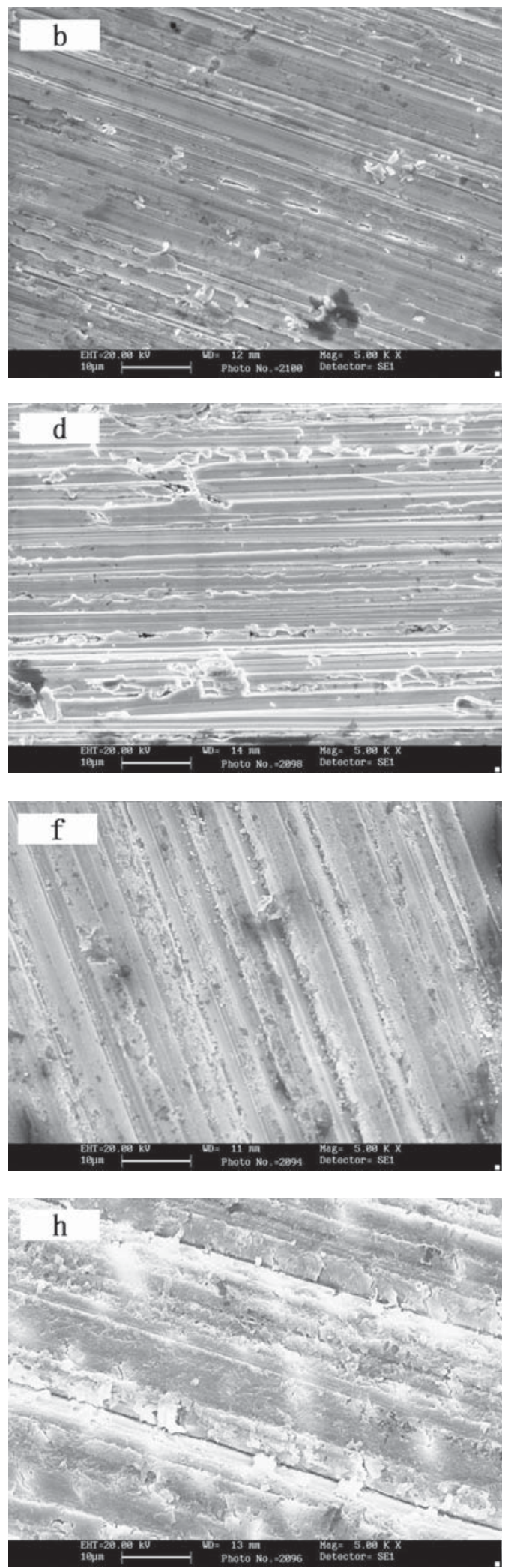

FIGURE 3- SEM images of bonding surfaces (x 5,000); (a) Nonprecious alloy treated with airborne-particle abrasion (Aa), (b) Nonprecious alloy treated with \#1000 SiC abrasive paper (Sp), (c) Precious alloy treated with Aa, (d) Precious alloy treated with Sp, (e) Enamel treated with Aa, (f) Enamel treated with Sp, (g) Dentin treated with $\mathrm{Aa}$, (h) Dentin treated with $\mathrm{Sp}$ 

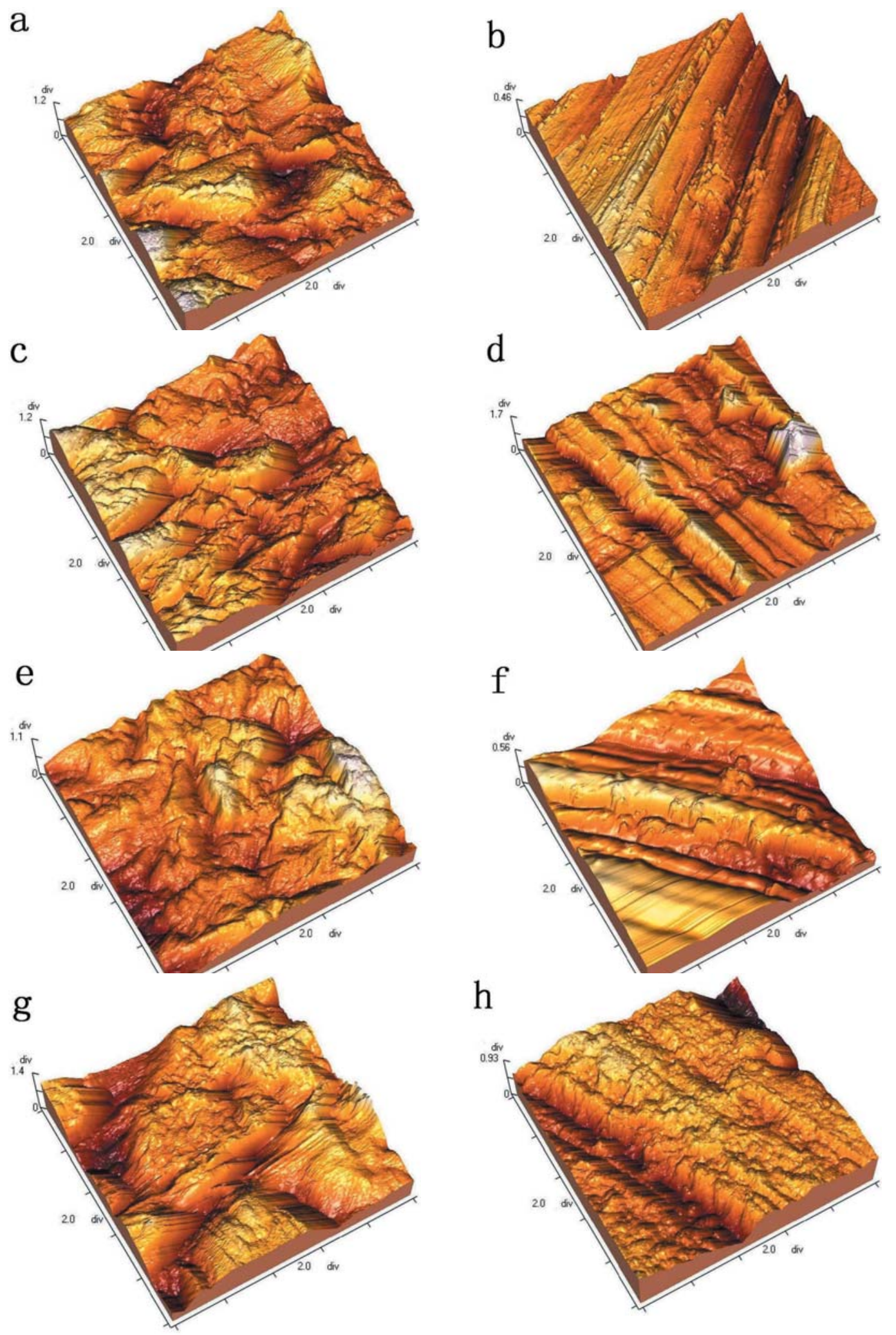

FIGURE 4- AFM 3D images of bonding surfaces ( $\times 5,000)$; (a) Nonprecious alloy treated with airborne-particle abrasion $(\mathrm{Aa})$, (b) Nonprecious alloy treated with \#1000 SiC abrasive paper (Sp), (c) Precious alloy treated with Aa, (d) Precious alloy treated with $\mathrm{Sp}$, (e) Enamel treated with Aa, (f) Enamel treated with Sp, (g) Dentin treated with $\mathrm{Aa}$, (h) Dentin treated with Sp 
the control group $(\mathrm{p}<0.05)$, but there was no significant difference in tensile strength among the HEMA-added groups $(\mathrm{p}>0.05)$.

\section{Shear Bond Strength}

The SBS of the experimental HAGICs with different HEMA contents are presented in Figure 2. The SBS values were obviously different due to the different bonding surfaces. Therefore, two-way ANOVA instead of three-way ANOVA that included the type of bonding surface was performed. Based on Scheffe's multiple-comparison test for the HEMA content, the homogenous subsets were as follows (p>0.05): Control, 10\%HEMA / 10\%HEMA, 20\%HEMA / 50\%HEMA, 30\% HEMA / 40\% HEMA for the nonprecious alloy; Control, 10\%HEMA / 20\%HEMA, 30\%HEMA, $50 \%$ HEMA, 40\%HEMA for the precious alloy; Control, 50\% HEMA, 10\% HEMA, 20\% HEMA / 50\% HEMA, 10\%HEMA, 20\%HEMA, 30\%HEMA, 40\%HEMA for the enamel; and 50\%HEMA / 40\%HEMA, Control, 30\% HEMA, 10\%HEMA / 30\%HEMA, 10\% HEMA, $20 \%$ HEMA for the dentin.

The SBS to the nonprecious alloy firstly increased and then decreased as the HEMA content increased for both of the Aa and Sp groups, and the maximum value was observed when the HEMA content was $40 \%$ (Figure 2a). The SBS to the precious alloy firstly increased and then decreased as the HEMA content increased for the Aa group, and the maximum value was observed when the HEMA content was $30 \%$. As for the Sp group, although the SBS increased when HEMA was added, it was not influenced by the amount of HEMA $(\mathrm{p}<0.05)$. Furthermore, the Sp group showed lower SBS than the Aa group when the HEMA content was the same (Figure 2b).

The SBS to enamel firstly increased and then decreased as the HEMA content increased for both of the Aa and Sp groups, and the maximum SBS value was observed when the HEMA content was $40 \%$ for the Aa group and $30 \%$ for the Sp group (Figure 2c). The SBS values to dentin were higher when the HEMA contents were relatively lower (10$20 \%$ ) than those bonded to other surfaces, and the Aa group always showed higher SBS than the Sp group. The SBS also firstly increased and then decreased as the HEMA content increased for both of the Aa and Sp groups, and the maximum value was reached when the HEMA content was $20 \%$ for the Aa group and $10 \%$ for the Sp group. When the HEMA content was 50\%, the SBS was even lower than that of the control group (Figure 2d).

\section{SEM and AFM Analyses of Bonding Surfaces}

The SEM images of the nonprecious alloy, precious alloy, enamel and dentin surfaces after the Aa and Sp treatments are presented in Figure 3. In all examined surfaces, the Aa groups showed irregular surfaces compared to the Sp groups. However, regular and linear scratches made by the SiC paper were observed in the Sp groups. Dentinal tubules were not observed in either the Aa or the Sp groups because of smear layer formation.

Three-dimensional surface images of the nonprecious alloy, precious alloy, enamel and dentin surfaces that were taken after the Aa and Sp treatments are presented in Figure 4. The Aa group was irregular, while the Sp group showed a relatively regular surface topography, similar to the results based on the SEM analysis. The average surface roughness values ( $\mathrm{Ra}$ ) of the surfaces after the Aa and Sp treatments are presented in Figure 5. Ra values in the Aa group were higher than those in the Sp group regardless of the bonding surface based on t-test $(\mathrm{p}<0.05)$.

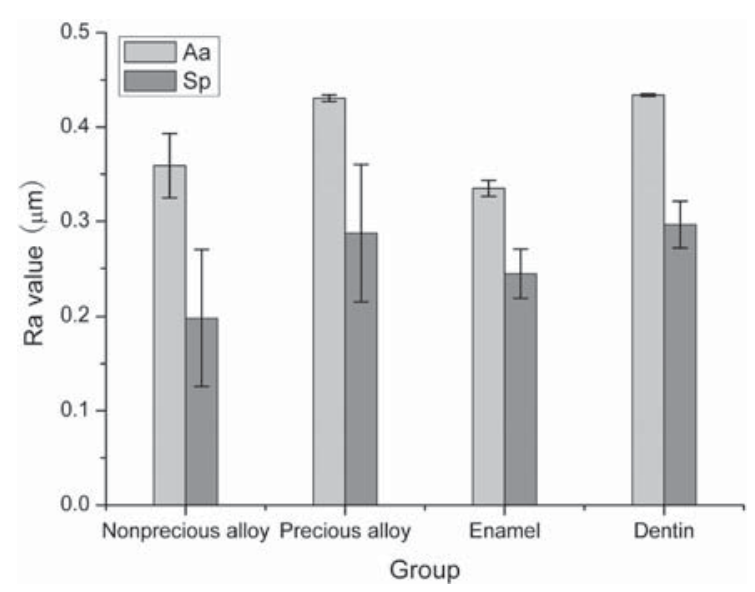

FIGURE 5- Ra value of bonding surfaces for the shear bond strength test

\section{DISCUSSION}

The first null hypothesis that there are no significant differences in the mechanical properties of the experimental HAGICs with increasing HEMA contents was rejected because both the compressive and diametral tensile strengths were influenced by the HEMA content $(\mathrm{p}<0.05)$. The second null hypothesis that there are no significant differences in the SBS of these HAGICs to dental alloys and teeth as a function of HEMA content was also rejected $(\mathrm{p}<0.05)$, except for the precious alloy.

RMGIC was developed to combine the advantages of conventional GIC and resin composite, and has been used for filling and bonding purposes ${ }^{2}$. The powder of a commercial RMGIC is composed of radiopaque fluoroaluminosilicate glass and a catalyst; its liquid component is composed of aqueous polyacrylate with a methacrylate group and also contains HEMA and tartaric $\operatorname{acid}^{10}$. Therefore, the setting reaction of this material is a combination of the acid-base reaction of conventional GIC and the polymerization reaction of methacrylate. Based on the analysis of the liquid component of commercial RMGICs, Ikeda, et al. ${ }^{10}$ showed that the HEMA content varies according to the commercial brand and assumed that modifications might have be done in the formulation of the RMGICs. In addition to the improvement of the physical 
properties of RMGICs by the addition of HEMA compared to conventional GICs, the low viscosity and low molecular weight of the RMGIC liquid also delay the setting reaction and improve the handling properties of RMGICs compared to those of conventional GICs ${ }^{10}$. However, based on these studies, it is impossible to clearly determine the influence of HEMA itself on the properties of RMGIC, which was the rationale for performing the present study.

In the present study, type II GIC (Fuji II) was used as the starting material. Since the viscosity restorative GICs is comparatively higher than that of luting GICs, a restorative GIC was selected because the viscosity of the liquid was expected to decrease after the addition of HEMA. In commercial materials, camphoroquinone and organic amines such as N,N-dimethylaminoethyl methacrylate are added to initiate the photoactivation of resins ${ }^{28}$. However, since the goal of the present study was to investigate the change in the mechanical properties of HAGICs according to the uncured HEMA content, other ingredients were not added in order to clearly evaluate the effect of HEMA. As used in commercial RMGICs, the addition of photoinitiator and coinitiator would make the experimental HAGICs to have similar properties to those of RMGICs. However, as light polymerization was not performed in the present study, even though the resultant HAGICs seemed to set like a hard solid, they most likely had lower mechanical properties than polymerized material because polymerization of HEMA was not intentionally performed. Polymerization of HEMA should be performed in a future study to elucidate the final effect of HEMA on resin-added GICs.

The effect on a water-activated glass polyacrylate cement of replacing the water with methanol or HEMA was studied ${ }^{1}$. The presence of the organic compounds caused the setting reaction to be substantially slowed down, and the resulting cements to be significantly weaker than those prepared with water. The use of water/HEMA had less effect on the speed of set than the use of water/methanol, but led to weaker cements. The post-hardening maturation processes that gradually increase the compressive strength of glass polyacrylate cements were found to take place in both water/ HEMA and water/methanol. In the present study, similar phenomena observed in HEMA-replaced GIC occurred. Neverthelles, direct comparison was not possible.

The compressive strength was measured $24 \mathrm{~h}$ after mixing the powder and liquid; the strength in the control group was $115.0 \mathrm{MPa}$ and changed to 112.3 and $112.8 \mathrm{MPa}$ in the $10 \%$ HEMA and $20 \%$ HEMA groups, respectively. From $30 \%$ HEMA on, the compressive strength decreased and reached $87.9 \mathrm{MPa}$ at 50\% HEMA. These results are similar to those of a previous study ${ }^{13}$ in which the compressive strength of the experimental RMGIC was lower than that of conventional GIC after $24 \mathrm{~h}$ of setting. This result might be caused by the lower compressive strength of HEMA, which was added to modify the high brittleness of GIC set by acid-base reaction for $24 \mathrm{~h}$.

Diametral tensile strength was also measured $24 \mathrm{~h}$ after setting in the present study. The strength of the control group specimens was $8.6 \mathrm{MPa}$, which gradually increased to the maximum value of $15.2 \mathrm{MPa}$ at $40 \%$ HEMA content and then decreased at 50\% HEMA content. These results are consistent with those of previous studies, which reported that the diametral tensile strength of experimental RMGICs was higher than that of conventional $\mathrm{GICs}^{13,23,26}$, although resin polymerization was not performed in the present study. It was possible to observe that as the HEMA content increased higher than $40 \%$, the diametral tensile strength decreased. Increased diametral tensile strength in the HEMA-added group seems to be related to the fact that the crack resistance increases due to the low elastic modulus of $\mathrm{HEMA}^{7}$. Light polymerization was performed in a previous study $^{7}$, but not in the present study. However, the results of both studies were similar, which might indicate that a small amount of acid-base reaction between glass particles of GIC and HEMA occurred in the present study.

The SBS values increased as the HEMA content increased up to certain content. After this point, the SBS decreased for all surfaces except for the precious alloy-Sp group. This trend was similar to that of the changes in diametral tensile strength. For most surfaces, the maximum SBS was observed when the HEMA content was 30-40\%. However, for both dentin-As and dentin-Sp groups, the maximum SBS was observed in the 20\% HEMA group. There was no change in SBS for the precious alloy-Sp group regardless of the HEMA content. This result indicates that the bond strength of the experimental HAGICs to the alloy is not directly correlated with the increase of mechanical properties of this material.

In the present study, direct comparison between different bonding surfaces was not performed because the influence of HEMA content on the SBS clearly differed according to the type of bonding surface, which may be the result of a combination of 1) a proprietary chemical bonding reaction with a surface that differs in chemical composition, and 2) the micromechanical bonding properties of HEMA.

There has been little consensus on the effect of surface treatments on the bonding of RMGIC. Airborne-particle abraded dentin showed lower bond strengths than SiC paperabraded dentin in a previous study ${ }^{21}$, while in another study ${ }^{16}$ airborne-particle abraded dentin and enamel showed higher bond strengths than their SiC paper-abraded counterparts. The major difference between these studies is that in the first study, after the airborne-particle abrasion, no rinsing was performed, while in the second study, a thorough water rinsing was performed for $30 \mathrm{~s}$ after airborne-particle abrasion. In the present study, no rinsing was performed after the airborne-particle abrasion following the methodology of the first study ${ }^{21}$. The SBS to airborneparticle abraded dentin was higher than those to the $\mathrm{SiC}$ paper-abraded group. In the first study, \#600 SiC paper was used, but in the present study, \#1000 SiC paper was used. Therefore, it was no possible to make a direct comparison of the results of both studies. Based on the results of the present study, although Ra values in the Aa group were higher than those in the Sp group regardless of the bonding surface based on t-test $(p<0.05)$, the influence of surface treatment on the SBS was bonding surface-dependent. Therefore, a 
general conclusion based only on the surface treatment, on the SBS, could not be drawn.

Other factors that can influence the bond strength to a airborne particle-abraded surface include air pressure, distance from the nozzle to the surface, shape and size of airborne-particles, abrasion time, and the diameter of nozzle ${ }^{3,16}$, all of which had a combined effect on the surface topography of the airborne-particle abraded surface. To investigate the surface topography, SEM analysis and Ra value measurement with AFM were performed in the present study. The airborne particle-abraded surfaces showed rough and irregular surfaces compared to the \#1000 SiC paperground surfaces, regardless of the bonding substance based on the result of the present study. However, it is not clinically relevant to polish the enamel and dentin specimens with \#1000 SiC paper since it is not likely to have a tooth surface analogous to \#1000 SiC grinding under clinical conditions. This was a limitation of the present study.

For the nonprecious alloy and enamel, there was no significant difference in the SBS between the two types of surface treating. This result indicates that the SBS between the HAGIC and nonprecious alloy and enamel is less dependent on micromechanical bond strength compared with the precious alloy and dentin, which also implies that the contribution of the chemical bonding property of GIC is stronger for the nonprecious alloy and enamel surfaces.

The theoretical degree of cure of resin monomers is $100 \%$. However, under clinical conditions it is generally regarded that $20-25 \%$ of unpolymerized monomers remain, which can cause pulp damage if they are released ${ }^{6}$. Based on a cytotoxicity assay, a previous found that the low biocompatibility of RMGIC was caused by the release of HEMA $^{18}$. Hamid, et al. ${ }^{9}$ have described the toxicity and hypersensitivity of released HEMA on pulp cells. Furthermore, another study reported that HEMA caused delayed hypersensitivity and contact dermatitis ${ }^{11}$. Therefore, thorough polymerization of RMGICs is required to minimize the release of unreacted $\mathrm{HEMA}^{9}$. In the present study, since the main focus was the influence of HEMA on the properties of HAGICs, polymerization of HEMA was not performed. The effect of polymerization should be determined to make this experimental HAGIC a clinically feasible material. Further studies to investigate this issue should be performed.

Based on the results of the present study, the incremental addition of uncured HEMA to conventional GIC resulted in increased diametral tensile strength and enhanced SBS bonded to dental alloys and tooth surfaces. As the degree of polymerization increases, polymerization shrinkage and heat release also increase ${ }^{19}$. Therefore, the correlation between biocompatibility and mechanical properties should be simultaneously considered. In addition, enhanced biocompatible filling materials with better mechanical properties should be developed.

\section{CONCLUSIONS}

The compressive, diametral tensile and shear bond strengths of experimental HAGICs were influenced by HEMA content added to the liquid component. The influence of surface treatments, such as airborne particle abrasion and SiC abrasive paper grinding, on the SBSs of HAGICs to the non-precious alloy and enamel was not significant. However, the SBSs of HAGICs to the precious alloy and dentin pretreated with airborne particle abrasion were higher than those to surfaces pretreated with $\mathrm{SiC}$ abrasive paper grinding. Although polymerization of HEMA itself was not performed in the present study, the obtained results suggest that the addition of the appropriate HEMA content to GIC can enhance the diametral tensile strength of this material as well as increase its bond strength to dental alloys and tooth structures. This indicates that polymerization of resin monomer could enhance the mechanical and bonding properties of this type of material. The optimal HEMA content was found to range between 20 and $40 \%$, within the limitations of the present study.

\section{REFERENCES}

1- Anstice HM, Nicholson JW. Studies on the setting of polyelectrolyte materials Part II. The effect of organic compounds on a glass poly(alkenoate) cement. J Mater Sci Mater Med. 1994;5:299-302.

2- Antonucci JM. Toughened glass-ionomer cements. Trends Tech Contemp Dent Lab. 1988;5:4.

3-Arici S, Ozer M, Arici N, Gencer Y. Effects of sandblasting metal bracket base on the bond strength of a resin-modified glass ionomer cement: an in vitro study. J Mater Sci Mater Med. 2006;17:253-8.

4- Braden M, Clarke RL, Nicholson J, Parker S. Polymeric dental materials. Berlin: Springer; 1997. p.1-50.

5- Cattani-Lorente MA, Dupuis V, Moya F, Payan J, Meyer JM. Comparative study of the physical properties of a polyacid-modified composite resin and a resin-modified glass ionomer cement. Dent Mater. $1999 ; 15: 21-32$.

6- Costa CAS, Hebling J, Hanks CT. Current status of pulp capping with dentin adhesive systems: a review. Dent Mater. 2000;16:188-97.

7- Eliades G, Palaghias G. In vitro characterization of visible light-cured glass ionomer liners. Dent Mater. 1993;9:198-203.

8- Gordan VV, Boyer D, Soderholm KJ. Enamel and dentin shear bond strength of two resin modified glass ionomers and two resin based adhesives. J Dent. 1998;26:497-503.

9- Hamid A, Okamoto A, Iwaku M, Hune WR. Component release from light-activated glass ionomer and compomer cements. J Oral Rehabil. 1998;25:94-9.

10- Ikeda K, Fujishima A, Suzuki M, Inoue M, Sasa R, Miyazaki T. Resin content in cement liquids of resin-modified glass ionomers. Dent Mater J. 1999;18:248-58.

11- Katsuno K, Manabe A, Itoh K, Nakamura Y, Wakumoto S, Hisamitsu $\mathrm{H}$, et al. Contact dermatitis caused by 2-HEMA and GM dentin primer solutions applied to guinea pigs and humans. Dent Mater J. 1996;15:2230 . 
12- Kobayashi M, Kon M, Miyai K, Asaoka K. Strengthening of glassionomer cement by compounding short fibers with $\mathrm{CaO}-\mathrm{P}_{2} \mathrm{O}_{2}-\mathrm{SiO}_{2}-\mathrm{Al}_{2} \mathrm{O}_{3}$ glass. Biomaterials. 2000;21:2051-8.

13- Mathis RS, Ferracane JL. Properties of glass ionomer/ resin-composite hybrid material. Dent Mater. 1989;5:355-8.

14- Mitra SB, inventor; Minnesota Mining \& MFG, assignee. Photocurable ionomer cement systems. European Patent Office EP 0323120A2, 1988 Dec 21.

15- Miyazaki M, Rikuta A, Iwasaki K, Ando S, Onose H. Influence of environmental conditions on bond strength of a resin-modified glass ionomer. Am J Dent. 1997;10:287-90.

16- Mujdeci A, Gokay O. The effect of airborne-particle abrasion on the shear bond strength of four restorative materials to enamel and dentin. J Prosthet Dent. 2004;92:245-9.

17- Nicholson JW, Anstice HM. The physical chemistry of light-curable glass-ionomer. J Mater Sci Mater Med. 1994;5:119-22.

18- Oliva A, Ragione FD, Salerno A, Riccio V, Tartaro G, Cozzolino A, et al. Biocompatibility studies on glass ionomer cements by primary cultures of human osteoblast. Biomaterials. 1996;17:1351-6.

19- Palmer G, Anstice HM, Pearson GJ. The effect of curing regime on the release of hydroxyethyl methacrylate (HEMA) from resin-modified glass-ionomer cements. J Dent. 1999;27:303-11.

20- Prosser HJ, Powis DR, Wilson AD. Glass-ionomer cements of improved flexural strength. J Dent Res. 1986;65:146-8.

21- Rinaudo PJ, Cochran MA, Moore BK. The effect of air abrasion on shear bond strength to dentin with dental adhesives. Oper Dent. 1997;22:254-9.

22- Rusz JE, Antonucci JM, Eichmiller F, Anderson MH. Adhesive properties of modified glass-ionomer cements. Dent Mater. 1992;8:31-6.

23- Sidhu SK, Watson TF. Resin-modified glass ionomer materials. Am J Dent. 1995;8:59-67

24- Simmons JJ. The miracle mixture glass ionomer and alloy powder. Tex Dent J. 1983;10:6-12.

25- Wilson AD. Resin-modified glass-ionomer cements. Int J Prosthodont. 1990;3:425-9.

26- Xie D, Brantley WA, Culbertson BM, Wang G. Mechanical properties and microstructures of glass-ionomer cements. Dent Mater. 2000;16:12938 .

27- Yap AUJ, Mudambi S, Chew CL, Neo JCL. Mechanical properties of an improved visible light-cured resin-modified glass ionomer cement. Oper Dent. 2001;26:295-301.

28- Yuhda S, Kameda H, inventors; Sankin Ind Co., assignee. Method of setting a dental cement composition. United States Patent and Trademark Office US 6063832. 1996 Oct 11 . 